\title{
Efficacy of recombinant human soluble thrombomodulin to treat disseminated intravascular coagulation
}

\author{
Kaname Uno*, Takuji Ueno, Takuma Yamada, Takehiko Takeda, Sho Tano, Masato Yoshihara, Michinori Mayama, Mayu Ukai, Teppei \\ Suzuki, Yasuyuki Kishigami and Hidenori Oguchi \\ Department of Obstetrics, Perinatal Medical Center, Toyota Memorial Hospital, Japan
}

\begin{abstract}
Background: Disseminated intravascular coagulation (DIC) treatment is essential for the management of placental abruption. Recombinant human soluble thrombomodulin (rhTM) is a novel anticoagulant that has antifibrinolytic and anti-inflammatory properties.

Case: A patient with severe DIC induced by placental abruption with fetal death was successfully treated with the early initiation of rhTM and discharged 2 days after delivery without any complications.

Conclusion: Early initiation of rhTM blocks further thrombin generation and can inhibit the coagulation cascade of DIC without increasing the risk of bleeding. rhTM also reduces secondary clinical consequences associated with endothelial damage and systemic inflammation because of antifibrinolytic and anti-inflammatory properties. rhTM administration is a promising therapeutic option for the management of DIC associated with placental abruption with fetal death.
\end{abstract}

\section{Introduction}

Placental abruption is a major cause of Disseminated Intravascular Coagulation (DIC), and in cases resulting in fetal death, the priority of treatment is to minimize maternal morbidity and mortality. Vaginal delivery with appropriate anti-DIC therapy is considered a first-line therapy, except when external hemorrhage cannot be controlled or when vaginal delivery is contraindicated because of obstetrical reasons [1].

In placental abruption, massive hemorrhage and activation of the extrinsic pathway by tissue factors cause the consumption and subsequent exhaustion of coagulation factors, resulting in DIC. Recombinant human soluble Thrombomodulin (rhTM) is a novel anticoagulant that is widely used for treatment of sepsis-induced DIC [2]. rhTM shows anticoagulant efficacy through reducing thrombinmediated clotting and enhancing protein $\mathrm{C}$ activation [3]. In addition, rhTM has antifibrinolytic and anti-inflammatory properties, which may prevent organ failure and hemorrhagic complications $[3,4]$. Here we report a case of placental abruption with fetal death that was successfully managed with appropriate plasma replacement therapy and rhTM administration. This strategy has vast potential for anti-DIC therapy in placental abruption.

\section{Case}

A 31-year-old Japanese woman (gravida 1, para 1) at 31 weeks and 1 day of gestation visited her antenatal clinic because of abdominal pain, genital bleeding, and intrauterine fetal death. Ultrasonography revealed broad retroplacental hematoma and no fetal heartbeat. She was subsequently transferred to our hospital $8 \mathrm{~h}$ after onset with a diagnosis of placental abruption. On physical examination, she appeared pale and her body temperature was $36.9^{\circ} \mathrm{C}$, regular pulse rate of 110 beats/ min, blood pressure of $107 / 77 \mathrm{mmHg}$, respiratory rate of 22 breaths/ min, and oxygen saturation of $100 \%$ (oxygen mask $6 \mathrm{~L} / \mathrm{min}$ ). Rigidity of abdomen muscles and broad purple spots on her limbs were noted.
Laboratory studies on admission revealed severe coagulopathy with a fibrinogen level of $<20 \mathrm{mg} / \mathrm{dL}$ (not detected), antithrombin III (AT-III) of $98 \%$, fibrin degradation products (FDP) of $>1200 \mu \mathrm{g} / \mathrm{mL}$, D-dimer of $>600 \mu \mathrm{g} / \mathrm{mL}$, activated partial thromboplastin time (aPTT) of 43.7 $\mathrm{s}$, and prothrombin time (PT) of $19.8 \mathrm{~s}$. White blood cell count was $13,800 / \mu \mathrm{L}$, platelet count was $86,000 / \mu \mathrm{L}$, and hemoglobin concentration was $8.9 \mathrm{~g} / \mathrm{dL}$. Other laboratory data were within normal ranges. Her International Society on Thrombosis and Haemostasis DIC score was 7 points (overt DIC).

Intensive DIC therapy was attempted with the transfusion of blood products and rhTM administration. The decision was made to administer rhTM at a dose of $380 \mathrm{IU} / \mathrm{kg}$ before using $12 \mathrm{U}$ of fresh frozen plasma (FFP), $2 \mathrm{U}$ of packed red blood cells (RBCs), and $10 \mathrm{U}$ of platelets. Vaginal delivery was selected because her vital signs were almost stable, and contraindications were absent. Because her cervical ostium was still closed, and uterine contractions were not apparent, we could not immediately rupture the membrane; therefore, labor was induced by administering incremental doses of oxytocin and inserting a double balloon catheter. Over the next $4 \mathrm{~h}$, her laboratory data significantly improved, with a fibrinogen level of $124 \mathrm{mg} / \mathrm{dL}$, AT-III of $91 \%$, FDP of $845.5 \mu \mathrm{g} / \mathrm{mL}$, D-dimer of $414.6 \mu \mathrm{g} / \mathrm{mL}$, aPTT of $31.2 \mathrm{~s}$, PT of $12.3 \mathrm{~s}$, white blood cell count of $8,800 / \mu \mathrm{L}$, platelet count of $96,000 /$ $\mu \mathrm{L}$, and hemoglobin concentration of $7.1 \mathrm{~g} / \mathrm{dL}$. At that time, the cervical ostium was $3 \mathrm{~cm}$ and amniotomy was performed. Over the next $3 \mathrm{~h}$, her laboratory values improved following a second transfusion of RBCs and FFP. The clinical course of this patient is shown in figure 1.

${ }^{*}$ Correspondence to: Kaname Uno, Department of Obstetrics, Perinatal Medical Center, Toyota Memorial Hospital, 1-1, Heiwa-cho, Toyota, Aichi, Japan, Tel: +81-565-28-0100, E-mail: kanjinkyaname@yahoo.co.jp

Received: May 10, 2018; Accepted: May 24, 2018; Published: May 28, 2018 


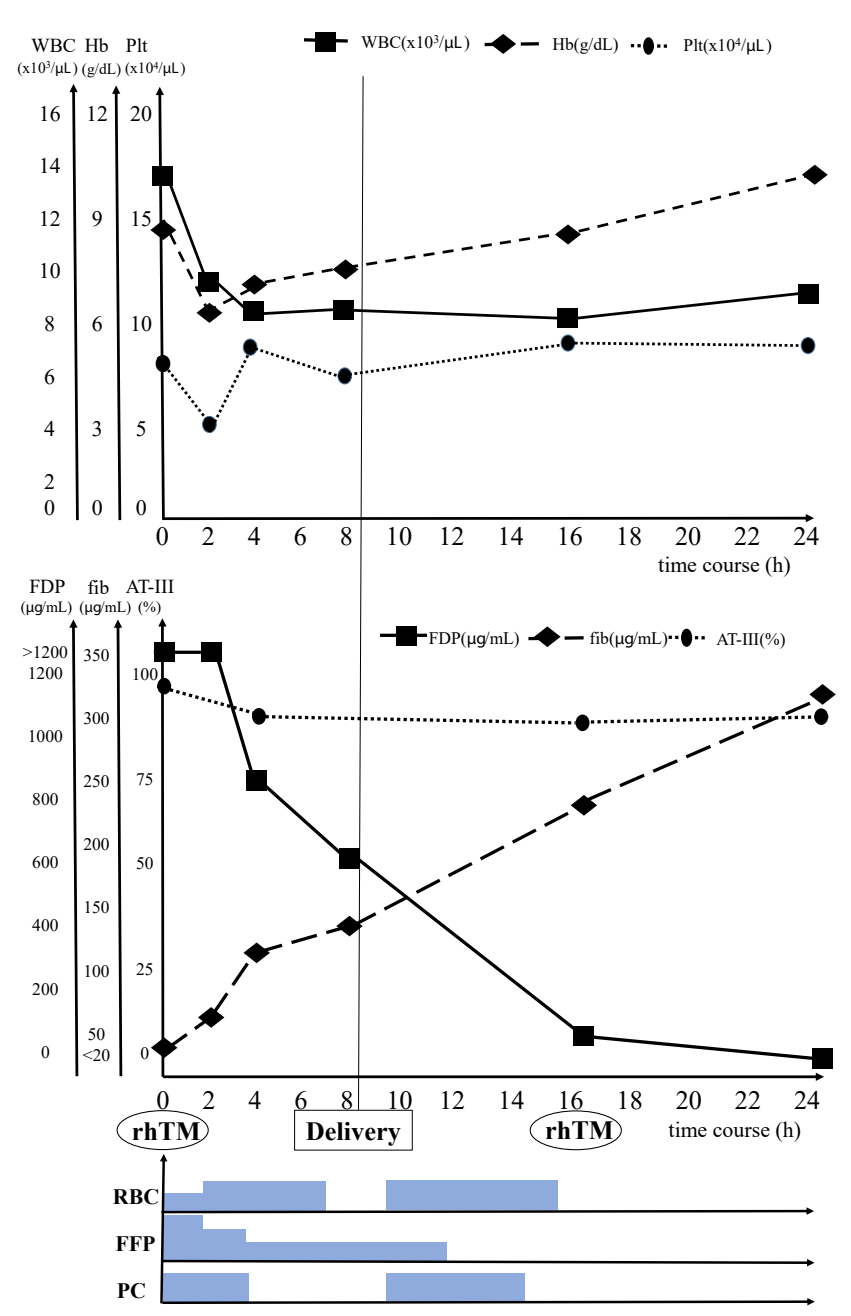

Figure 1. The clinical course of the patient after arrival. With rhTM administration and plasma replacement, a significant reduction in FDP, increase fibrinogen level and sustained AT- III activity were confirmed.

After over $16 \mathrm{~h}$ from placental abruption onset, a dead fetus and placenta were extracted. The delamination area was approximately $60 \%$. After delivery, oxytocin was administered, and bimanual compression of her uterus was performed. The total amount of hemorrhage was $3,741 \mathrm{~g}$, and the total volume of blood transfused was $18 \mathrm{U}$ of packed RBCs, $32 \mathrm{U}$ of FFP, and $35 \mathrm{U}$ of platelets. After delivery, there was no indication of renal, hepatic, pulmonary, or cardiac failure. There was no evidence of pathologic thrombosis. She was discharged from the hospital in a stable condition on postdelivery day 2.

\section{Discussion}

In the present report, we describe a case of a patient who underwent vaginal delivery after placental abruption resulting in fetal death. Intensive DIC therapy with early initiation of rhTM and labor stimulation with amniotomy was thought to be the key factors for the accomplishment of the delivery. To the best of our knowledge, this is the first report of the clinical use of rhTM for obstetrical DIC caused by placental abruption with fetal death and successful vaginal delivery. Therefore, rhTM administration may be suitable for the management of DIC associated with placental abruption with fetal death.

The anticoagulant efficacy of rhTM has been described in DIC, which is characterized by uncontrolled and excessive thrombin production [4]. Thrombomodulin, in vitro, inhibits further thrombin generation and inactivates fibrinogen cleavage, leading to the suppression of subsequent microvascular thrombus formation [5]. Moreover, the administration of rhTM, which decreases the interactions between thrombin and fibrinogen, can directly decrease fibrinogen-tofibrin degradation. Furthermore, the reaction between thrombin and thrombomodulin is reversible and faster than that between thrombin and antithrombin [6], resulting decreased thrombin and antithrombin reaction rates without compromising antithrombin function. In addition, thrombin-rhTM complexes enhance protein $\mathrm{C}$ activation, which serves as a negative regulator of the coagulation pathway through the inactivation of the cofactors Va and VIIIa [3]. This complex is also known to have antifibrinolytic and anti-inflammatory effects [5], differentiating rhTM from other anticoagulants. These properties can mediate the relatively profibrinolytic status arising from consumption coagulopathy without provoking hemorrhagic complications and improve systemic inflammation, resulting from the DIC status, where excessive activation of coagulation induces proteases that produce extensive endothelial damage and multiple organ failure [4]. In our patient, a significant reduction in FDP concentration and sustained AT-III activity were confirmed after rhTM administration and plasma replacement. DIC might be mediated by rhTM via the inhibition of extreme thrombin generation and suppression of profibrinlytic and pro-inflammatory activities.

Placental abruption is a significant cause of obstetric DIC and is associated with increased maternal and fetal morbidity and mortality. Decidual bleeding and hypoxia yield the release of tissue factors, which generate an excessive amount of thrombin, leading to the expression of inflammatory cytokines and hypercoagulation that overwhelms hemostatic control mechanisms, followed by DIC [7]. Early rhTM administration not only improves the procoagulant state through its ability to inhibit thrombin but also reduces secondary clinical consequences associated with endothelial damage and systemic inflammation. Particularly for patients with placental abruption and fetal death, the immediate initiation of rhTM is logically reasonable. In the absence of other contraindications, vaginal delivery is the preferable option in such cases as long as the mother's condition is stable [1]. With labor stimulation by immediate rupture of the membrane, intensive DIC treatment is essential for the completion of vaginal delivery, which can lead to quick recovery and subsequent successful pregnancy and delivery, as demonstrated in our patient [8].

In conclusion, rhTM can be a key drug for the treatment for DIC in the obstetrical field. Further accumulation of cases and clinical trials are needed to establish the effectiveness and safety of rhTM for the management of vaginal delivery in cases of placental abruption with fetal death.

\section{Acknowledgements}

I would like to express my sincere gratitude to Dr. Jintetsu Minami, who had supported the management of our case with patience and knowledge. I also wish to express my appreciation to Takehiko Takeda, Sho Tano, Mayu Ukai, Yasuyuki Kishigami. They contributed to elaborating our manuscript and gave us considerable encouragements.

\section{References}

1. Oylese Y, Ananth CV (2006) Placental abruption. Obstet Gynecol 108: 1005-1016. [Crossref]

2. Vincent JL, Ramesh MK, Ernest D, LaRosa SP, Pachl J, et al. (2013) A randomized, double-blind, placebo-controlled, Phase $2 b$ study to evaluate the safety and efficacy of recombinant human soluble thrombomodulin, ART-123, in patients with sepsis and suspected disseminated intravascular coagulation. Crit Care Med 41: 2069-2079. [Crossref] 
Uno K (2018) Efficacy of recombinant human soluble thrombomodulin to treat disseminated intravascular coagulation

3. Mohri M, Sugimoto E, Sata M, Asano T (1999) The inhibitory effect of recombinant human soluble thrombomodulin on initiation and extension of coagulation--a comparison with other anticoagulants. Thromb Haemost 82: 1687-1693. [Crossref]

4. Levi M (2004) Current understanding of disseminated intravascular coagulation. $\mathrm{Br} \mathrm{J}$ Haematol 124: 567-576. [Crossref]

5. Martin FA, Murphy RP, Cumminus PM (2013) Thrombomodulin and the vascular endothelium: insights into functional, regulatory, and therapeutic aspects. Am J Physiol Heart Circ Physiol 304: 1585-1597. [Crossref]
6. Aritomi M, Watanabe N, Ohishi R, Gomi K, Kiyota T, et al. (1993) Recombinant human soluble thrombomodulin delivers bounded thrombin to antithrombin III thrombomodulin associates with free thrombin and is recycled to activate protein $\mathrm{c}$. Thromb Haemost 70: 418-422. [Crossref]

7. Thachil J, Toh CH (2009) Disseminated intravascular coagulation in obstetric disorders and its acute haematological management. Blood Rev 23:167-176. [Crossref]

8. Imanaka S, Naruse K, Akasaka J, Shigematsu A, Iwai K, et al. (2014) Vaginal delivery after placental abruption and intrauterine fetal death, including failed cases. Int $J$ Gynaecol Obstet 126: 180-181. [Crossref]

Copyright: $(02018$ Uno K. This is an open-access article distributed under the terms of the Creative Commons Attribution License, which permits unrestricted use, distribution, and reproduction in any medium, provided the original author and source are credited. 